\title{
Measurement of morphological parameters of giant cell tumor of bone in the knee
}

\author{
LIN GUO $^{1,2}$, PENG JIA ${ }^{3}$, YONG-CHENG HU ${ }^{4}$, MAN SUN $^{1}$, ZHI WANG $^{1}$, XUE-NING ZHANG ${ }^{2}$ and JUN MIAO ${ }^{4}$ \\ ${ }^{1}$ Department of Radiology, Tianjin Hospital; ${ }^{2}$ Department of Radiology, The Second Hospital of Tianjin Medical University; \\ Departments of ${ }^{3}$ Orthopaedics and ${ }^{4}$ Bone Tumors, Tianjin Hospital, Tianjin 300211, P.R. China
}

Received June 15, 2018; Accepted January 23, 2019

DOI: $10.3892 / \mathrm{ol} .2019 .10064$

\begin{abstract}
The aim of the present study was to characterize the morphological parameters of giant cell tumor of bone (GCTB) in the knee. The imaging data of 250 patients with GCTB in the knee were retrospectively reviewed, and the morphological parameters were analyzed. The study included 137 cases with GCTB in the distal femur and 113 cases with GCTB in the proximal tibia. The maximal longitudinal diameter of the tumor was $6.616 \pm 2.322 \mathrm{~cm}$ in the femur group and $5.738 \pm 2.278 \mathrm{~cm}$ in the tibia group $(\mathrm{P}=0.003)$. The maximal transverse diameter in the two groups was $4.865 \pm 1.525$ and $4.313 \pm 1.309 \mathrm{~cm}$, respectively $(\mathrm{P}=0.003)$. The shortest distance from the articular surface (SDAS) in the two groups was $0.381 \pm 0.404$ and $0.280 \pm 0.328 \mathrm{~cm}$, respectively $(\mathrm{P}=0.035)$, whereas the longest distance from the articular surface in the two groups was $6.924 \pm 2.135$ and $5.878 \pm 1.825 \mathrm{~cm}$, respectively $(\mathrm{P}=0.001)$. There were statistically significant differences between the two groups in terms of the range of SDAS $(\mathrm{P}=0.043)$. Additionally, the incidence of pathological fractures in the femur was higher compared with that in the tibia $(\mathrm{P}=0.001)$, and the incidence of pathological fractures in the two groups gradually increased with the increase in lesion diameter. GCTB in the distal femur was larger compared with that in the proximal tibia, whereas GCTB in the tibia was closer to the articular surface compared with that in the femur. Furthermore, the incidence of pathological fractures in the femur was higher compared with that in the tibia.
\end{abstract}

Correspondence to: Dr Yong-Cheng Hu, Department of Bone Tumors, Tianjin Hospital, 406 Jiefang South Road, Tianjin 300211, P.R. China

E-mail: yongchenghu_dr@163.com

Dr Xue-Ning Zhang, Department of Radiology, The Second Hospital of Tianjin Medical University, 23 Pingjiang Road, Tianjin 300211, P.R. China

E-mail: xueningzhang_dr@163.com

Key words: giant cell tumor of bone, knee joint, radiography imaging, morphology

\section{Introduction}

Giant cell tumor of bone (GCTB) is benign in histology, but presents as a local invasive growth, and its biological behavior is hard to predict (1). Although it is not a fatal tumor, pulmonary metastasis easily occurs in young patients with recurrent GCTB at Enneking stage 3 (2). GCTB is characterized by expansive growth and usually occurs at the end of a long bone, and in $>50 \%$ patients, it occurs at the knee-joint (3). In addition, the tumor tissue often approaches the articular surface by damaging bones covered by articular cartilage (4). Curettage can be used to remove the tumor without damaging the joint function, but a high recurrence rate following surgery remains a difficult problem to solve (5). Although the wide excision of tumors combined with bone tumor prosthesis can markedly decrease the recurrence rate, the function of the reconstructed joint is worse, when compared with the function of the joint following curettage, due to joint defects caused by wide excision (6-8). The range of osteotomy is correlated with tumor recurrence and joint reconstruction $(9,10)$. Therefore, investigating the morphology of GCTB may assist in the accurate excision of tumors, thus decreasing recurrence caused by residual tumor cells. A major problem encountered with GCTB is that the range of excision is restricted due to limitations on bone tumor prosthesis, which increases the risk of failing to reconstruct the joint. The use of personalized prosthesis for GCTB decreases unnecessary excision and allows for the retention of more healthy bone. The morphological changes of GCTB negatively affect the biomechanical property of bones and increase the risk of pathological fractures, which may influence the surrounding soft tissue and lead to tumor recurrence following surgery $(5,11,12)$. Therefore, morphological characteristics, including the size of the tumor, the distance of the tumor edge from the articular surface and occurrence of pathological fractures, have an important impact on the treatment of GCTB and on estimating prognosis.

The present study summarizes the morphological features of GCTB in the knee and provides evidence to support the use of these characteristics to improve the accuracy of tumor excision, to decrease damage to the host bone and improve the survival rate of prostheses. In addition, the present study provides a theoretical basis for predicting the occurrence of pathological fractures in GCTB and for designing a personalized GCTB prosthesis for the knee. 


\section{Materials and methods}

Inclusion and exclusion criteria. Inclusion criteria: i) Patients with tumors around the knee confirmed as GCTB by pathology; ii) patients with complete imaging data, including $\mathrm{X}$-ray films covering the whole area of the lesion, computed tomography (CT) scanning images in Digital Imaging and Communications in Medicine (DICOM) format or multi-dimensional magnetic resonance imaging (MRI); and iii) cases in which the morphological measurement of GCTB was not influenced by surgery. Exclusion criteria: i) Patients without a definite pathological diagnosis; ii) patients without complete clinical and imaging data; iii) patients with imaging data that was not in DICOM format; and iv) cases in which the imaging data did not cover the whole area of the lesion.

On the basis of the above criteria, a total of 255 patients who were diagnosed with GCTB by pathological surgery between June 2000 and December 2016 in eight hospitals were included in the present study. These hospitals are institutional members of the Chinese Giant Cell Tumor Team of China (GTOC)-associated group, including Tianjin Hospital (Tianjin, China), Jinan Military Region General Hospital (Jinan, China), Xijing Hospital (Xian, China), The Second Affiliated Hospital of Zhejiang University School of Medicine (Hangzhou, China), Nanjing Military Region General Hospital (Nanjing, China), West China Hospital of Sichuan University (Chengdu, China), The Second Hospital of Tianjin Medical University (Tianjin, China) and The Third Hospital of Hebei Medical University (Shijiazhuang, China). The clinical and imaging data of these 255 patients were retrospectively analyzed. Of these patients, 5 had GCTB in the proximal fibula $(2.0 \%)$. As GCTB rarely occurs in the proximal fibula, and the fibula bears no weight, these 5 cases were excluded from the present study. The present study involving 250 patients was conducted in accordance with The Declaration of Helsinki, and approved by the Medical Ethics Committee of Tianjin Hospital. Written informed consent was obtained from all participants and the patients gave permission for publication of their images.

Morphological parameters and measurement of the GCTB lesion. The morphological parameters of GCTB included the following: i) Maximal longitudinal diameter (MLD) of the tumor defined by the maximal diameter of the tumor parallel with the femur and tibia; ii) maximal transverse diameter (MTD) of the tumor defined by the maximal diameter of the tumor vertical or almost vertical with the femur and tibia; iii) the shortest and longest distance from the articular surface (SDAS and LDAS, respectively) defined as the shortest or longest distance of the tumor edge from the articular surface under the cartilage; and iv) the presence or absence of pathological fractures. It should be noted that the thickness of the articular cartilage did not require measurement. CT images of the coronal/sagittal reconstruction or MRI images of the coronal/sagittal plane with the maximal diameter were selected to measure the MLD, MTD, SDAS and LDAS of the tumors.

All these parameters were measured by two observers who had $>10$ years of experience in radiology, and were trained in the standards prior to observation and measurement of the

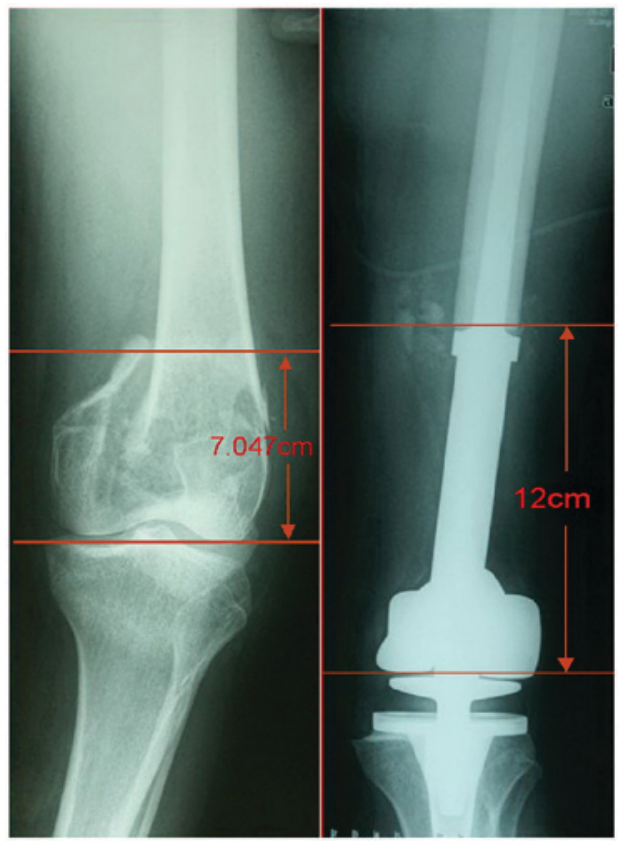

Figure 1. X-ray images of left distal femur in a 53-year-old male patient with a giant cell tumor of the bone. The longitudinal diameter of the tumor was $7.047 \mathrm{~cm}$ (right), and the length of the reconstruction was $12 \mathrm{~cm}$ to adjust to the prosthesis (left).

current samples, including determining the optimal measuring plane and point.

Statistical analysis. SPSS (version 19.0; IBM Corp., Armonk, NY, USA) was used to analyze the data. The intra-group correlation coefficient (ICC) was evaluated to compare the measurements of MLD, MTD, SDAS and LDAS from the two observers. Age, morphological parameters, and other parameters were expressed as the mean \pm standard deviation, and these data were compared using an independent sample t-test. A $\chi^{2}$ test was used to compare the enumeration data between two groups, such as the packet count data of SDAS and the incidence of pathological fractures between these two groups. $\mathrm{P}<0.05$ was considered to indicate a statistically significant difference.

\section{Results}

Clinical parameters. A total of 250 patients with GCTB were enrolled in the present study. The age range of the patients was 17.2-78.5 years, and the average age was $35.3 \pm 12.4$ years. Among the patients, $133(53.2 \%)$ were male and $117(46.8 \%)$ were female. Additionally, 137 patients $(54.8 \%)$ had GCTB in the distal femur, whereas $113(45.2 \%)$ had GCTB in the proximal tibia. The sex distribution of patients with GCTB in the distal femur was 73 male and 64 female, and the sex distribution of patients with GCTB in the proximal tibia was 62 male and 51 female. The difference in sex distribution was not significant, using a $\chi^{2}$ test. The average age of patients with GCTB in the distal femur was $35.6 \pm 12.7$ years, and the average age of patients with GCTB in the proximal tibia was $34.8 \pm 13.2$ years. There was no significant difference in the age of patients with GCTB between these two groups $(t=0.639, P=0.523)$. According to the imaging features, all patients presented with typical manifestations of GCBT, including expansive growth, osteolytic destruction and 


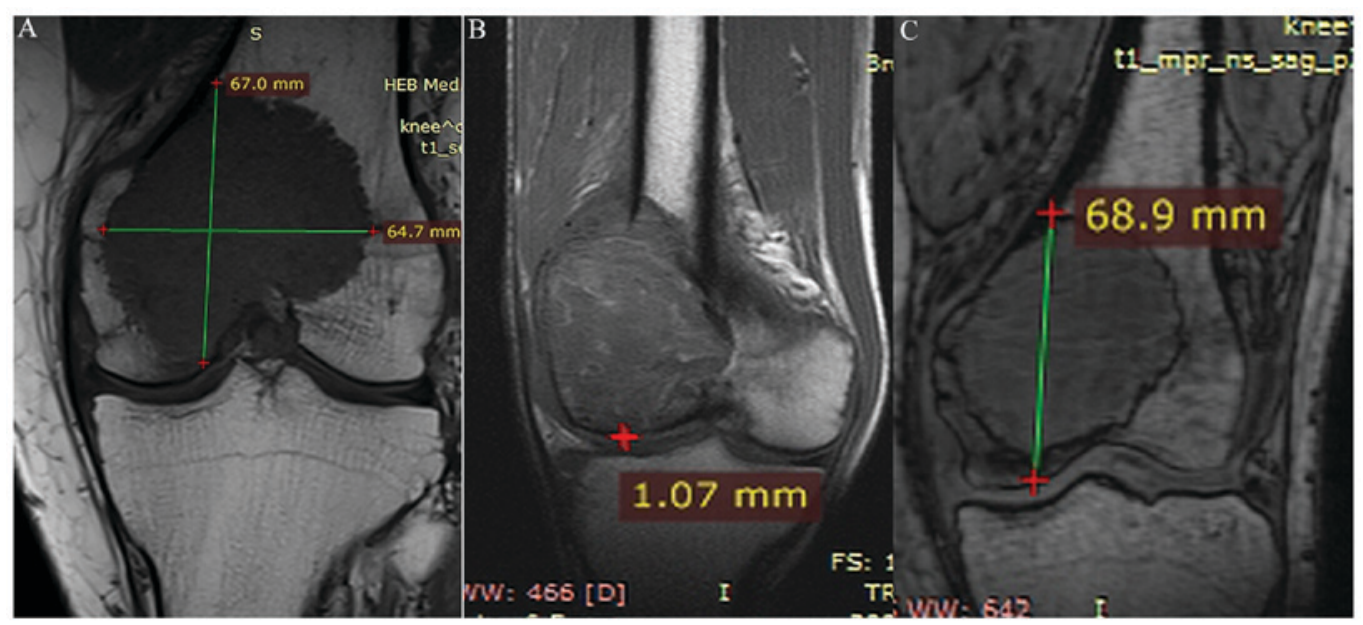

Figure 2. T1-weighted magnetic resonance images of a giant cell tumor in the distal femur of a 37-year-old male. (A) The maximal longitudinal diameter was $67.0 \mathrm{~mm}$ and the maximal transverse diameter was $64.7 \mathrm{~mm}$; (B) the shortest distance between the tumor edge and articular surface was $1.07 \mathrm{~mm}$; and (C) the longest distance between the tumor edge and the articular surface was $68.9 \mathrm{~mm}$.

no calcification. Of the patients, 187 were treated with tumor curettage and 63 underwent wide excision of tumors combined with bone tumor prosthesis (Fig. 1).

Intra-group consistency of the two observers. The morphological parameters were measured by two observers. For patients with GCTB in the distal femur, the ICC $r$ values of the MLD, MTD, SDAS and LDAS measurements were 0.9797, 0.9760, 0.9728 and 0.9650 , respectively, whereas those in patients with GCTB in the proximal tibia were $0.9971,0.9720,0.9605$ and 0.9935 , respectively. These results indicate that there was high intra-group consistency in the measurements of the two observers.

Measurement of the morphological parameters. Morphological parameters of GCTB were measured from the MRI/CT images (Fig. 2). The results of the measured morphological parameters were as follows.

$M L D$. Mean MLD in the group with GCTB in the distal femur was $6.616 \pm 2.322 \mathrm{~cm}$, and MLD in the group with GCTB in the proximal tibia was $5.738 \pm 2.278 \mathrm{~cm}$. The $95 \%$ confidence interval (CI) of MLD in the distal femur group was $6.222-7.008 \mathrm{~cm}$, and that in the proximal tibia group was $5.313-6.163 \mathrm{~cm}$. The measurements of MLD were significantly different between the two groups $(t=2.999, \mathrm{P}=0.003)$. The frequency distribution of MLD in the two groups demonstrates that the MLD measurement was generally normally distributed, with the exception of a number of tumors in the distal femur that were $>12 \mathrm{~cm}$, and a number in the proximal tibia that were $>10 \mathrm{~cm}$ (Fig. 3). In addition, $80 \%$ of the MLD measurements of tumors in the distal femur were $4.4-8.9 \mathrm{~cm}$, and $80 \%$ of the MLD values in the proximal tibia were $4.1-7.1 \mathrm{~cm}$.

$M T D$. The average MTD of tumors in patients with GCTB in the distal femur was $4.865 \pm 1.525 \mathrm{~cm}$, and that in patients with GCTB in the proximal tibia was $4.313 \pm 1.309 \mathrm{~cm}$. The $95 \%$ CI of MTD in patients with GCTB in the distal femur was $4.607-5.122 \mathrm{~cm}$, whereas that in patients with GCTB in the proximal tibia was 4.069-4.557 cm. There was a significant difference in MTD measurements between the two groups $(\mathrm{t}=3.232, \mathrm{P}=0.003)$. In patients with GCTB in the distal femur, $80 \%$ of MTD values were $3.1-6.4 \mathrm{~cm}$, whereas $80 \%$ of the MTD values in patients with GCTB in the proximal tibia were $2.7-6.0 \mathrm{~cm}$.

LDAS. The average LDAS in patients with GCTB in the distal femur was $6.924 \pm 2.135 \mathrm{~cm}$, and that in patients with GCTB in the proximal tibia was $5.878 \pm 1.825 \mathrm{~cm}$. The $95 \%$ CI of LDAS in patients with GCTB in the distal femur was $6.563-7.284 \mathrm{~cm}$, and that in patients with GCTB in the proximal tibia was 5.537-6.217 cm. There was a significant difference in LDAS between the two groups $(\mathrm{t}=4.116, \mathrm{P}=0.001)$. The histogram of the frequency distribution of LDAS in the two groups was similar to the histograms of MLD and MTD, and was normally distributed (data not shown). In patients with GCTB in the distal femur, $80 \%$ of the LDAS values were within $4.9-9.2 \mathrm{~cm}$, whereas in patients with GCTB in the proximal tibia, $80 \%$ of the LDAS values were within $4.4-7.5 \mathrm{~cm}$.

SDAS. The average SDAS in patients with GCTB in the distal femur was $0.381 \pm 0.404 \mathrm{~cm}$, and that in patients with GCTB in the proximal tibia was $0.280 \pm 0.328 \mathrm{~cm}$. The $95 \%$ CI of SDAS in patients with GCTB in the distal femur was $0.313-0.449 \mathrm{~cm}$, and that in patients with GCTB in the proximal tibia was $0.219-0.342 \mathrm{~cm}$. There was a significant difference in SDAS between the two groups $(\mathrm{t}=2.211, \mathrm{P}=0.035)$. The histogram of the frequency distribution of SDAS in the two groups demonstrates that lower SDAS measurements were more frequent (Fig. 4). In patients with GCTB in the distal femur, $80 \%$ of the SDAS values were within $0.01-0.75 \mathrm{~cm}$, whereas in patients with GCTB in the proximal tibia, $80 \%$ of the SDAS values were within $0.01-0.51 \mathrm{~cm}$.

Grouping statistics of SDAS. To further evaluate the association between the distance from the tumor edge and the articular surface and the location of the tumor, all subjects were divided into five ranges on the basis of the SDAS values: 0-1, 1-3, 3-6, 6-10 and >10 $\mathrm{mm}$ (Table I). These results indicate that there was a significant difference between the two groups when SDAS was $0-1 \mathrm{~mm}\left(\chi^{2}=9.837, \mathrm{P}=0.043\right)$. The majority of 

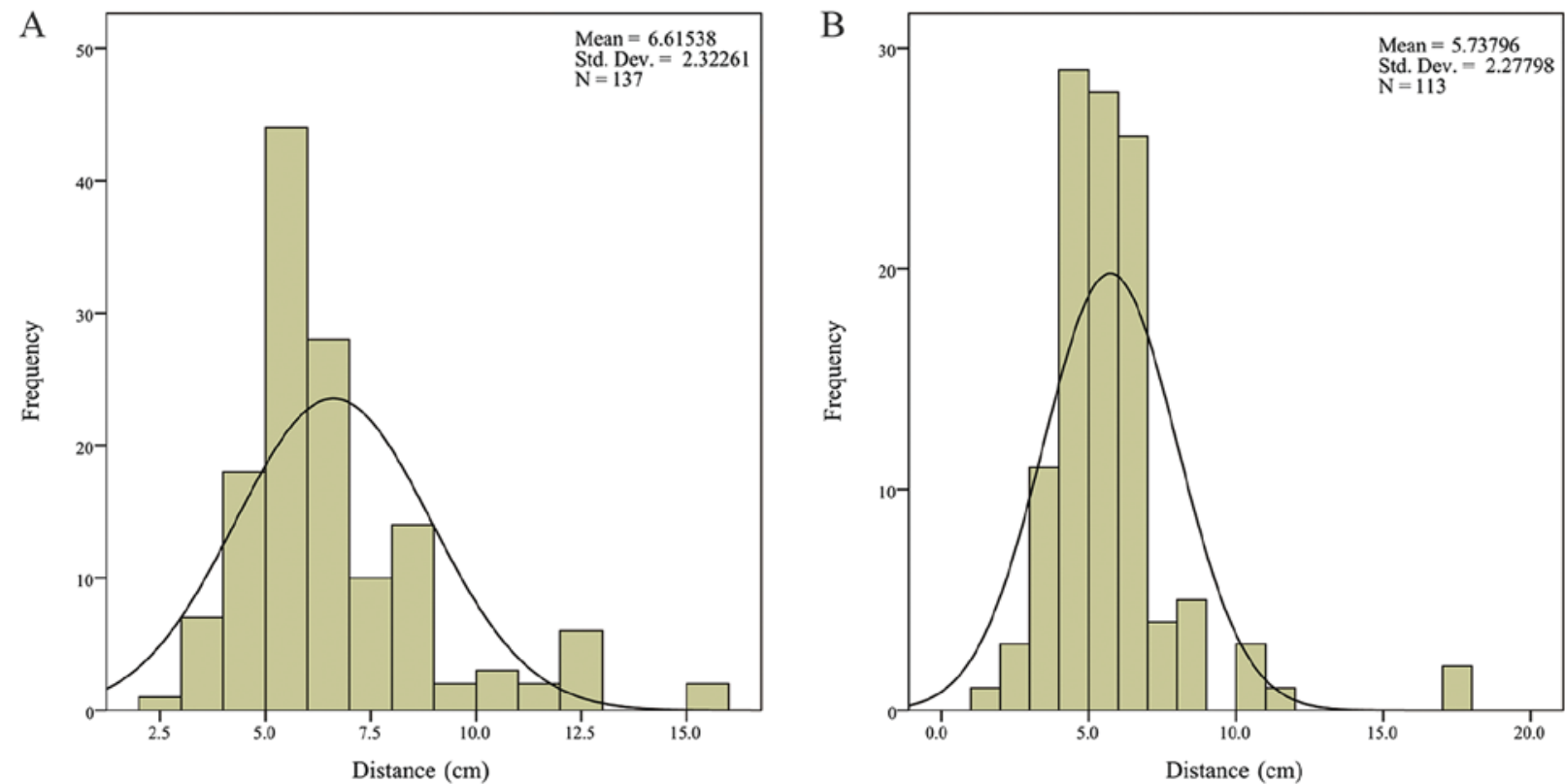

Figure 3. Frequency distribution of maximal longitudinal diameter of the giant cell tumors of the bone (A) in the distal femur and (B) in the proximal tibia.
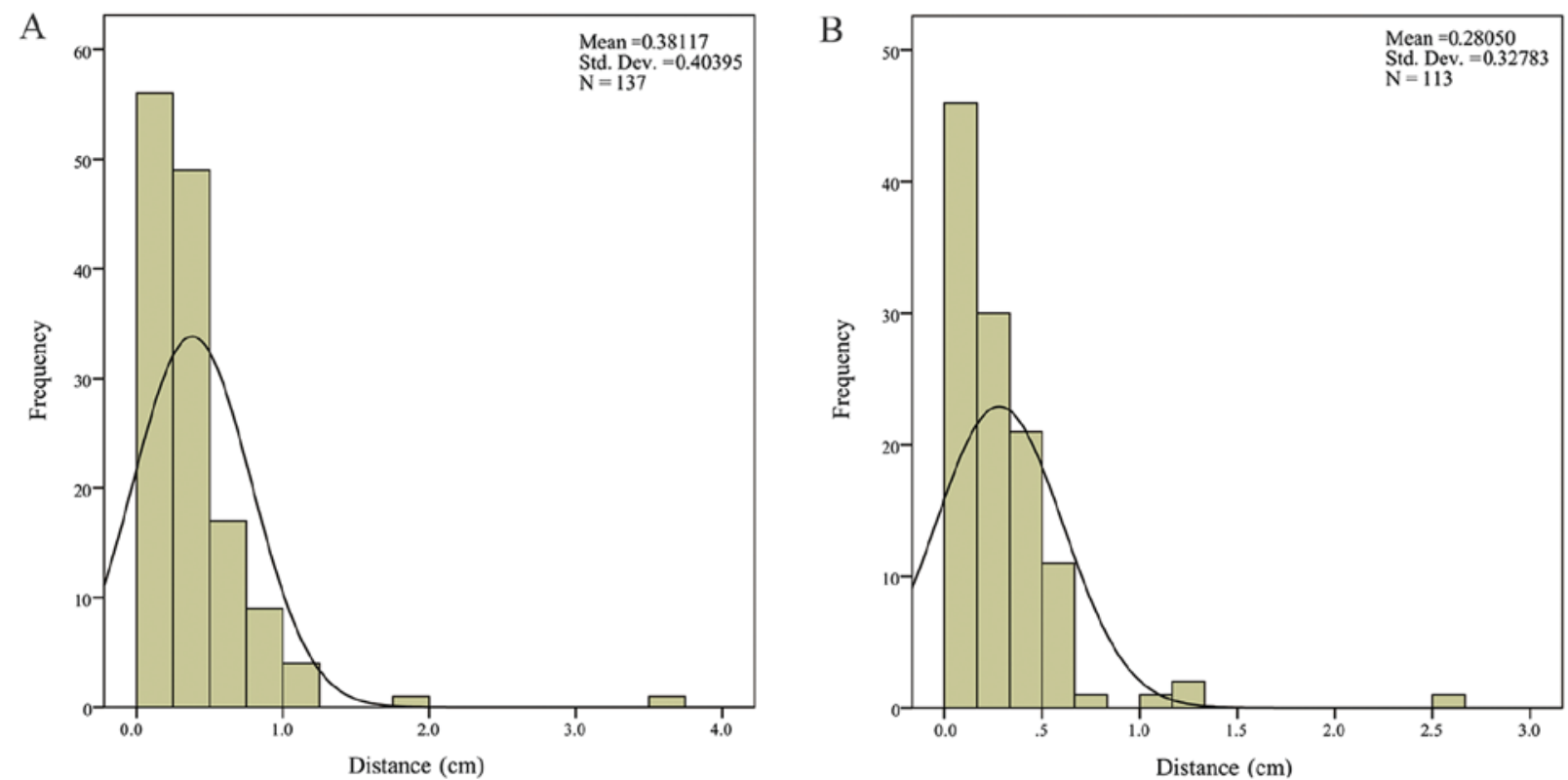

Figure 4. Frequency distribution of the shortest distance from the articular surface in patients with giant cell tumors of the bone (A) in the distal femur, and (B) in the proximal tibia.

patients in the two groups fell into the range 0-3 $\mathrm{mm}$ (66 cases in the distal femur and 69 cases in the proximal tibia). Therefore, SDAS presented a markedly skewed distribution in the two groups. The edge of the tumor in the two groups was evidently close to the articular surface. The further the tumor edge was from the articular surface, the lower the frequency.

Association between the size of the tumor and incidence of pathological fractures. A total of 64 patients (25.1\% of 255) presented with pathological fractures. Of those patients, 47 patients had GCTB in the distal femur (34.4\% of 137 patients with GCTB in the distal femur), whereas 17 patients had GCTB in the proximal tibia (15.0\% of 113 patients with GCTB in the proximal tibia). There was a significant difference in the incidence of pathological fractures between the two groups $(\mathrm{P}=0.001)$. The MLD was divided into different ranges, with intervals of $3 \mathrm{~cm}$. With the exception of the $0-3 \mathrm{~cm}$ range, the incidence of pathological fractures in the distal femur was higher compared with that in the proximal tibia (Table II). The MTD was divided into ranges with $2 \mathrm{~cm}$ intervals. For all ranges, the incidence of pathological fractures was higher in the distal femur compared with that in the proximal tibia (Table III). The trend of pathological fracture incidence in Tables II and III indicated that the frequency of fracture increased with the increase in longitudinal and transverse diameter in the two groups. 
Table I. Range of SDAS measurements of giant cell tumors of the bone in the distal femur and in the proximal tibia.

\begin{tabular}{|c|c|c|c|c|c|c|c|}
\hline \multirow[b]{2}{*}{ Location of tumor } & \multicolumn{5}{|c|}{ SDAS, mm } & \multirow[b]{2}{*}{$\chi^{2}$} & \multirow[b]{2}{*}{ P-value } \\
\hline & $0-1$ & $1-3$ & $3-6$ & $6-10$ & $>10$ & & \\
\hline Distal femur, n (\%) & $23(16.9)$ & $43(31.4)$ & $37(27.0)$ & $18(13.1)$ & $16(11.6)$ & 9.837 & 0.043 \\
\hline Proximal tibia, n (\%) & 35 (30.9) & $34(30.0)$ & $29(25.6)$ & $9(8.1)$ & $6(5.4)$ & & \\
\hline
\end{tabular}

SDAS, shortest distance from the articular surface.

Table II. Incidence of pathological fractures and range of MLD of giant cell tumors of the bone in the distal femur and in the proximal tibia.

\begin{tabular}{|c|c|c|c|c|c|c|c|}
\hline \multirow[b]{2}{*}{ Location of tumor } & \multicolumn{5}{|c|}{ MLD, cm } & \multirow[b]{2}{*}{$\chi^{2}$} & \multirow[b]{2}{*}{ P-value } \\
\hline & $0-3$ & $3-6$ & $6-9$ & $>9$ & Total & & \\
\hline Distal femur, n/total (\%) & $0 / 3(0.0)$ & 23/71 (32.4) & $21 / 57(36.8)$ & $3 / 6(50.0)$ & $47 / 137(34.3)$ & 12.063 & 0.001 \\
\hline Proximal tibia, n/total (\%) & $1 / 5(20.0)$ & 8/67 (12.0) & $6 / 35(17.1)$ & $2 / 6(33.0)$ & $17 / 113(15.0)$ & & \\
\hline
\end{tabular}

MLD, maximal longitudinal diameter.

Table III. Incidence of pathological fractures and range of MTD of giant cell tumors of bone in the distal femur and in the proximal tibia.

\begin{tabular}{|c|c|c|c|c|c|c|c|c|}
\hline \multirow[b]{2}{*}{ Location of tumor } & \multicolumn{6}{|c|}{ MTD, cm } & \multirow[b]{2}{*}{$\chi^{2}$} & \multirow[b]{2}{*}{ P-value } \\
\hline & $0-2$ & $2-4$ & $4-6$ & $6-8$ & $>8$ & Total & & \\
\hline Distal femur, $\mathrm{n} /$ total (\%) & $0 / 1(0.0)$ & $10 / 37(27.0)$ & $27 / 78(34.6)$ & $8 / 18(44.4)$ & $2 / 3(66.7)$ & 47/137 (34.3) & 12.063 & 0.001 \\
\hline Proximal tibia, n/total (\%) & $0 / 3(0.0)$ & $6 / 44(13.6)$ & $7 / 50(14.0)$ & $4 / 15(26.7)$ & $0 / 1(0.0)$ & $17 / 113(15.0)$ & & \\
\hline
\end{tabular}

MTD, maximal transverse diameter.

\section{Discussion}

The aim of treating GCTB is to improve knee function as much as possible following surgery, while also ensuring that the risk of tumor recurrence is decreased. A GCTB with a large diameter can cause extensive bone cortical damage, and induce damage in a large surrounding soft tissue mass and pathological fractures, or extensive invasion to the articular surface. These types of tumor should be treated with segmental resection and reconstruction, in order to remove the tumor and maintain knee function (12). Therefore, accurate measurement of the size and range of the tumor is essential to improve the success rate of surgery (9). If the tumor lesion is not sufficiently excised, there is a high risk of recurrence (10). Alternatively, if the tumor lesion is excessively excised, the normal host bone may be damaged and limb function may be affected. Therefore, similar to other bone tumors, the morphological features of GCTB, including size, distance from the articular surface and pathological fractures, are important factors that influence the surgical method and reconstruction of the tumor cavity, and the prognosis.
The extent of surgical excision required is decided on the basis of the length of the GCTB and the length of the prosthesis in the reconstruction. Therefore, a definite length of the longitudinal diameter of the GCTB would assist when devising a surgical plan, and in determining the plane in which the bone should be cut. Kivioja et al (13) measured the morphological parameters of GCTB in a group of patients with tumors in long tubular bones, and reported that the mean tumor length was $5 \mathrm{~cm}$. However, that cohort included tumors in upper and lower limbs, the sample size was small, and a single method of measurement was used. The present study had a large sample size and focused on GCTB in the knee, and therefore avoided introducing variation due to differences in growth characteristics and biological behavior of GCTB in different locations. Hu (14) measured the length of GCTB in 60 patients in the knee using MRI, and reported that the mean length of the lesion was $6.7 \mathrm{~cm}$, and the range was 4-12 cm. However, tumors in the femur and tibia were not investigated separately. In the present study, the mean longitudinal diameter of tumor in the distal femur was similar to that identified in the study by $\mathrm{Hu}$ (14). However, for tumors in the proximal tibia, the mean longitudinal diameter in the present 
study was markedly smaller compared with that in the study by $\mathrm{Hu}$ (14). Furthermore, the mean longitudinal diameter of tumors in the present study was higher compared with that observed by Kivioja et al (13), indicating that the longitudinal diameter of GCTB in the knee may be higher compared with that in the humerus, radius and other locations. Therefore, the range of osteotomy and the reconstructed length in the knee may be bigger compared with that in other locations. Szendröi (15) identified that in $\sim 1 / 3$ of patients, the size of the tumor was $>50 \%$ of the diameter of the host bone at the initial diagnosis. The present study revealed that the mean MTD of GCTB in the distal femur was $>50 \%$ of the diameter of the pulp cavity of the distal femur. Thus, the mechanical strength of the distal femur may be easily damaged, and pathological fractures easily occur.

The clinical significance of the distance of the tumor edge from the articular surface was recognized in previous studies. Ward and Li (16) suggested that the surgical excision plan should be influenced by the degree by which the GCTB has invaded the articular surface. In a retrospective study conducted by Blackley et al (17) on 59 cases with GCTB in the long bone, an association between the distance of the tumor edge from the articular surface and the incidence of postoperative articular degeneration was identified. In the present study, the mean shortest distance of GCBT in the knee from the articular surface was $<5 \mathrm{~mm}$. Patients with GCTB in the femur at a distance of 0-3 $\mathrm{mm}$ from the articular surface accounted for $48.2 \%$ (66/137 cases), and those with tumors in the tibia at that distance accounted for $61 \%$ (68/113 cases). This indicates that the majority of GCTB cases in the knee may occur close to the articular surface.

Dreinhofer et al (18) reported 15 cases of GCTB with pathological fractures. The distance between the tumor and articular surface was observed by measuring the thickness of the subchondral bone, and in 8 cases $(53.3 \%)$ this measurement was $0 \mathrm{~mm}$. The present study identified a statistically significant difference in the SDAS of GCTB in the distal femur and proximal tibia, suggesting that GCTB in the proximal tibia could more easily invade the articular surface under the soft bone, when compared with that in the distal femur. Thus, collapse of the articular surface and articular degeneration more easily occurred in the patients with GCTB in the proximal tibia. Therefore, in treating GCTB of the proximal tibia, more attention should be given to articular integrity.

Pathological fractures increase the complexity of the surgery required to completely remove tumors. Therefore, understanding the association between the incidence of pathological fractures and tumor morphology is important to determining treatment of GCTB. In a retrospective study of 54 cases of distal femur GCTB, Jeys et al (19) identified that $22 \%$ of the 12 cases had pathological fractures. The present study revealed that the incidence of pathological fractures in GCTB in the distal femur (34.4\%) was higher compared with that reported by Jeys et al (19), and compared with that in the proximal tibia (15.0\%). The incidence of fracture in the femur and tibia groups increased as the tumor diameter increased. In addition, the present study revealed that GCTB diameter in the distal femur was larger compared with that in the proximal tibia, which may explain the increased incidence of pathological fractures in the distal femur compared with that in the proximal tibia.
Owing to the lack of studies on morphological parameters of GCTB and the accurate measurement of the size of the tumor, for early GCTB excision, the range of the tumor lesion is determined by X-ray plus the addition of $3-5 \mathrm{~cm}$ to the border of the osteotomy to avoid recurrence caused by residual tumor cells (14). However, in increasing the length of reconstruction, the risk of complications with the prosthesis increases. If the size of the tumor is determined prior to surgery, an accurate osteotomy can be conducted. Thus, the healthy host bone can be preserved as much as possible, decreasing the risk of complications with the prosthesis. This may assist in increasing the service life of the prosthesis and developing a personalized prosthesis in the future. Chen et al (20) performed a multicenter retrospective study on 42 patients with distal femoral GCTB, who underwent artificial prosthesis replacement. It was demonstrated that the length of the osteotomy was significantly associated with loosening of the prosthesis, and decreased flexion and extension function of the knee, particularly when the osteotomy length was $>12 \mathrm{~cm}$. As the number of patients included in the present and previous studies is small, more patients are required to develop a database of morphological parameters of GCTB in the knee and other sites, in order to provide a theoretical basis for the diagnosis and treatment of GCTB.

\section{Acknowledgements}

Not applicable.

\section{Funding}

No funding was received.

\section{Availability of data and materials}

The datasets used and/or analyzed during the current study are available from the corresponding author on reasonable request.

\section{Authors' contributions}

YCH and XNZ designed the study. YCH, LG and JM analyzed and interpreted the patient data. PJ, MS, ZW and JM collected the patient data, and LG, YCH and XNZ were major contributors in writing the manuscript. All authors read and approved the final manuscript.

\section{Ethics approval and consent to participate}

This study was approved by the Medical Ethics Committee of Tianjin Hospital (Tianjin, China).

\section{Patient consent for publication}

Written informed consent was obtained from all participants, and the patients gave permission for publication of their images.

\section{Competing interests}

The authors declare that they have no competing interests. 


\section{References}

1. Traub F, Singh J, Dickson BC, Leung S, Mohankumar R, Blackstein ME, Razak AR, Griffin AM, Ferguson PC and Wunder JS: Efficacy of denosumab in joint preservation for patients with giant cell tumour of the bone. Eur J Cancer 59: 1-12, 2016.

2. Chan CM, Adler Z, Reith JD and Gibbs CP Jr: Risk factors for pulmonary metastases from giant cell tumor of bone. J Bone Joint Surg Am 97: 420-428, 2015.

3. Sobti A, Agrawal P, Agarwala S and Agarwal M: Giant cell tumor of bone-an overview. Arch Bone Jt Surg 4: 2-9, 2016.

4. Fraquet N, Faizon G, Rosset P, Phillipeau JM, Waast D and Gouin F: Long bones giant cells tumors: Treatment by curretage and cavity filling cementation. Orthop Traumatol Surg Res 95: 402-406, 2009.

5. Klenke FM, Wenger DE, Inwards CY, Rose PS and Sim FH: Giant cell tumor of bone: Risk factors for recurrence. Clin Orthop Relat Res 469: 591-599, 2011.

6. Zhang S, Zhang J and Wang X: Comparison of tumor curettage and resection for treatment of giant cell tumor of the bone around the knee joint. Pak J Med Sci 32: 662-666, 2016.

7. Balke M, Schremper L, Gebert C, Ahrens H, Streitbuerger A, Koehler G, Hardes J and Gosheger G: Giant cell tumor of bone: Treatment and outcome of 214 cases. J Cancer Res Clin Oncol 134: 969-978, 2008.

8. Boons HW, Keijser LC, Schreuder HW, Pruszczynski M, Lemmens JA and Veth RP: Oncologic and functional results after treatment of giant cell tumors of bone. Arch Orthop Trauma Surg 122: 17-23, 2002.

9. Shin KH, Park HJ, Yoo JH and Hahn SB: Reconstructive surgery in primary malignant and aggressive benign bone tumor of the proximal humerus. Yonsei Med J 41: 304-311, 2000.

10. Dürr HR, Maier M, Jansson V, Baur A and Refior HJ: Phenol as an adjuvant for local control in the treatment of giant cell tumour of the bone. Eur J Surg Oncol 25: 610-618, 1999.

11. Deheshi BM, Jaffer SN, Griffin AM, Ferguson PC, Bell RS and Wunder JS: Joint salvage for pathologic fracture of giant cell tumor of the lower extremity. Clin Orthop Relat Res 459: 96-104, 2007.
12. van der Heijden L, Dijkstra PD, Campanacci DA, Gibbons CL and van de Sande MA: Giant cell tumor with pathologic fracture: Should we curette or resect? Clin Orthop Relat Res 471: 820-829, 2013.

13. Kivioja AH, Blomqvist C, Hietaniemi K, Trovik C, Walloe A, Bauer HC, Jorgensen PH, Bergh P and Follerås G: Cement is recommended in intralesional surgery of giant cell tumors: A scandinavian sarcoma group study of 294 patients followed for a median time of 5 years. Acta Orthop 79: 86-93, 2008.

14. Hu YC: Related prognostic factors in surgical treatment of giant cell tumor of the knee around the knee. Chin J Orthop 32: 1083-1090, 2012 (In Chinese).

15. Szendröi M: Giant-cell tumour of bone. J Bone Joint Surg Br 86: 5-12, 2004.

16. Ward WG Sr and Li G III: Customized treatment algorithm for giant cell tumor of bone: Report of a series. Clin Orthop Relat Res: 259-270, 2002.

17. Blackley HR, Wunder JS, Davis AM, White LM, Kandel R and Bell RS: Treatment of giant-cell tumors of long bones with curettage and bone-grafting. J Bone Joint Surg Am 81: 811-820, 1999.

18. Dreinhöfer KE, Rydholm A, Bauer HC and Kreicbergs A: Giant-cell tumours with fracture at diagnosis. Curettage and acrylic cementing in ten cases. J Bone Joint Surg Br 77: 189-193, 1995.

19. Jeys LM, Suneja R, Chami G, Grimer RJ, Carter SR and Tillman RM: Impending fractures in giant cell tumours of the distal femur: Incidence and outcome. Int Orthop 30: 135-138, 2006.

20. Chen GJ, Wang Z, Wang L, Hu YC, Yu XC, Ye ZM, Wu SJ, Zhang GC and Guo SB: A multicenter retrospective study of artificial joint replacement on giant cell tumor in distal femur. Chin J Orthop 38: 338-345, 2018.

This work is licensed under a Creative Commons Attribution-NonCommercial-NoDerivatives 4.0 International (CC BY-NC-ND 4.0) License. 